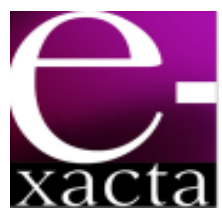

ISSN: 1984-3151

\title{
ANÁLISE DO SINAL WI-FI NO ESTACIONAMENTO DO AEROPORTO INTERNACIONAL TANCREDO NEVES - MG
}

\author{
ANALYSIS OF WI-FI SIGNAL IN THE PARKING LOT OF THE MG - \\ TANCREDO NEVES INTERNATIONAL AIRPORT
}

\author{
Arlete Vieira da Silva; Euzébio D. de Souza; \\ Flávio Rodrigues Santos; Guilherme Teófilo Reis Siuves
}

Centro Universitário de Belo Horizonte, Belo Horizonte, MG

arlete.silva@prof.unibh.br, euzebio.souza@prof.unibh.br, flaviobeaga@yahoo.com.br, vortice@oi.com.br

Recebido em: 28/06/2011 - Aprovado em: 06/07/2011 - Disponibilizado em: 24/07/2011

RESUMO: Este documento apresenta uma técnica para analisar a intensidade do sinal Wi-Fi das redes locais sem fio. Esse método tem como objetivo encontrar redes wireless em um ambiente outdoor (aberto) e analisá-las através da metodologia Site Survey. Um estudo de caso é conduzido no estacionamento do Aeroporto Internacional Tancredo Neves-MG, e os resultados são apresentados georreferenciados à planta baixa do local. PALAVRAS-CHAVE: Aeroporto. Estacionamento. NetStumbler. Site Survey. Wireless. Wi-Fi.

ABSTRACT: This paper presents a technique for analyzing the signal strength of Wi-Fi wireless local area networks. This method aims to find wireless networks in an outdoor environment (open) and analyze them through the Site Survey methodology. A case study is conducted in the parking lot of the Tancredo Neves International Airport and the results are presented georeferenced to the floor plan of the site.

KEYWORDS: Airport, Parking, NetStumbler, Site Survey, Wireless, Wi-Fi.

\section{INTRODUÇÃO}

As redes sem fio se tornaram uma boa opção de comunicação entre computadores nos dias de hoje. Essas redes operam de maneira similar às redes cabeadas, com a diferença de que não há cabos ligando os computadores da rede. A grande vantagem de uma rede wireless é a mobilidade, ou seja, o usuário estando com seu notebook em uso, por exemplo, pode se movimentar livremente ao longo de uma área, não estando restrito a um local fixo como nas redes cabeadas (FARIAS, 2007).

Atualmente, uma das principais tecnologias wireless destinadas à transmissão de dados entre dispositivos computacionais é a Wi-Fi, cujo nome é originado do termo Wireless Fidelity (fidelidade sem fios). Esta tecnologia tornou-se a de mais rápida adoção no mundo wireless dos últimos anos e já se encontra em muitos dispositivos móveis, como os palmtops e notebooks, como consta em (JARDIM, 2007).

O termo Wi-Fi é utilizado para descrever os produtos que aderem aos padrões 802.11.x criado pelo IEEE (Institute of Electrical and Electronics Engineers). Para PINHEIRO (2003) os padrões mais conhecidos são o 802.11b, para dispositivos que operam na banda de $2,4 \mathrm{GHz}$ e que permite transferir dados com taxas de $11 \mathrm{Mbps}$ e o padrão $802.11 \mathrm{~g}$ que é compatível com o 802.11b e com o padrão 802.11a. O padrão "a" descreve dispositivos que operam na banda de $5 \mathrm{GHz}$ com taxas até $54 \mathrm{Mbps}$. 
De acordo com (FARIAS, 2006), uma WLAN, ou rede local, pode ser utilizada tanto na forma indoor quanto na forma outdoor. É dito que uma WLAN é indoor quando o sinal está sendo transmitido em ambiente fechado, normalmente na presença de muitos obstáculos, um escritório é um bom exemplo e possui pequeno alcance (até 300 metros). Já uma WLAN é outdoor quando o sinal está sendo transmitido ao ar livre, por exemplo: uma comunicação entre dois prédios. As antenas ficam nos topos dos prédios e para que haja comunicação é necessário haver visada direta entre elas. Essas antenas captam sinais de longo alcance podendo chegar a vários quilômetros.

Como qualquer tecnologia de rede, as wireless também encontram obstáculos para implantação de sua estrutura. As WLAN's sofrem com obstáculos ou interferências ambientais, onde as peculiaridades do espaço podem atenuar a potência do sinal Wi-Fi, reduzindo, por conseqüência, a taxa de transferência e a qualidade da conexão, assim como a viabilidade de alguns serviços que exigem um limite mínimo de predicados para funcionar com eficiência.

Este estudo se propõe a analisar e apresentar um método simplificado capaz de rastrear e registrar a intensidade do sinal Wi-Fi em um ambiente outdoor. Comumente essa metodologia é chamada de Site Survey (VAUGHAN-NICHOLS, 2003), que poderia ser traduzido como "Inspeção do local" e tem por objetivo permitir ao usuário encontrar redes sem fio, medir níveis de potência do sinal de ruído e identificar os endereços físicos(MAC) da estação transmissora (Pontos de Acesso).

Um estudo de caso é conduzido em ambiente outdoor, e o local escolhido para este trabalho foi o estacionamento do Aeroporto Internacional Tancredo Neves.

\section{Estudo de CAso}

O Aeroporto Internacional Tancredo Neves encontrase na Região Metropolitana de Belo Horizonte, no município de Confins, antigo distrito do município de Lagoa Santa (Minas Gerais).

Amplo, moderno e funcional, o Aeroporto Internacional Tancredo Neves é o principal portal de entrada para o Estado de Minas Gerais e foi projetado para cinco milhões de passageiros por ano. Atualmente existe uma previsão de adequações no terminal atual de passageiros (orçado em $\mathrm{R} \$ 7$ milhões) para ampliar sua capacidade de 5 para 7 milhões de passageiros/ano, como preparação para a copa de 2014, segundo site de informações aeroportodeconfins.wordpress.com (WORDPRESS 2011).

A Figura 1 mostra uma foto área do aeroporto de Confins.

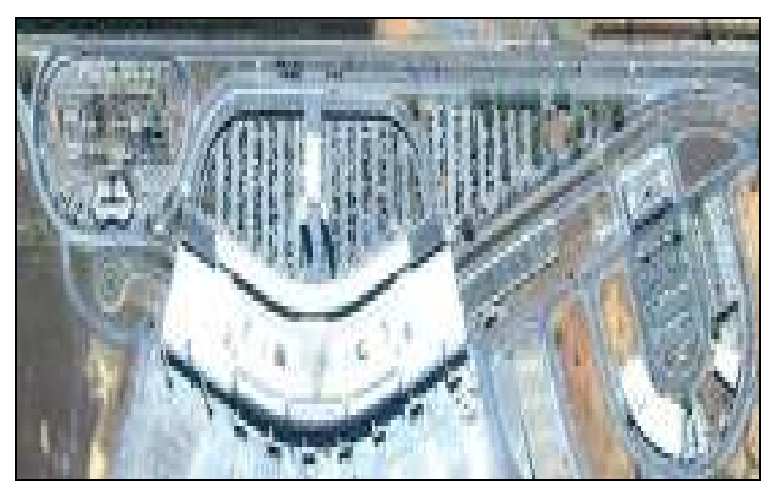

FIGURA 1. Imagem via satélite do Aeroporto de Confins.

FONTE: GOOGLE MAPS, 2011

Para atender a um público cada vez mais exigente e constantemente conectado, a Infraero disponibilizou em 2005 a infra-estrutura de rede local de comunicação de dados nos aeroportos para fornecimento de conexão sem fio à internet.

A tecnologia escolhida para ser utilizada no aeroporto de Confins foi a Wi-Fi (Wireless Fidelity, ou fidelidade sem fio) e a empresa escolhida para a prestação desses serviços foi a VEX (Pointer Networks $S / A$ ), que 
atualmente é a líder e pioneira no mercado Wi-Fi no Brasil (INFRAERO, 2011).

No estacionamento foram instaladas duas antenas do tipo painel, localizadas na torre frontal do aeroporto, onde cada torre possui aproximadamente 26 metros de altura. Na Figura 2 pode-se observar a localização das antenas painéis em uma dessas torres.

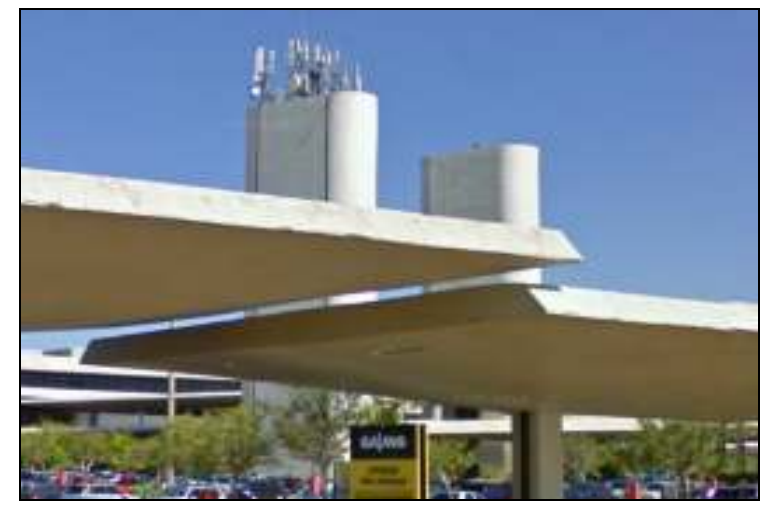

FIGURA 2. Imagem das antenas instaladas na torre frontal do Aeroporto de Confins.

FONTE: GOOGLE MAPS, 2011.

As duas antenas instaladas no estacionamento do aeroporto são do modelo Ubiquit Nanostation e operam na freqüência de $2.4 \mathrm{GHZ}$, com $12 \mathrm{Dbi}$ de ganho.

O formato da antena foi especificado pela infraestrutura do aeroporto para que o hotstpot (ponto de acesso) abrangesse somente o estacionamento e parte da área de check-in. A Figura 3 é um exemplo de antena tipo painel.

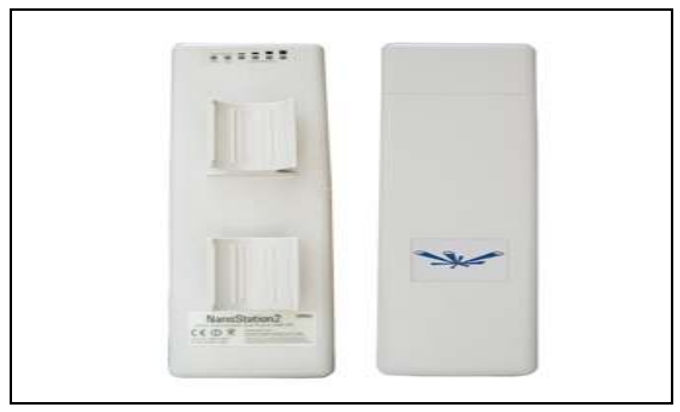

FIGURA 3. Exemplo de uma antena painel.

FONTE: www.linkteck.com.br, 2011
Cada antena atende a somente um lado do estacionamento, já que ela é do tipo painel e, também, para que a onda eletromagnética irradiada pela antena esteja direcionada do lado oposto à área de embarque e da torre de controle para que não cause interferências na rede wireless do aeroporto (INFRAERO,2011).

\section{Metodologia}

A pesquisa se caracteriza como um estudo de caso em um ambiente outdoor através da técnica comumente conhecida como Site Survey, onde a melhor localização é obtida após a análise e cruzamento dos dados de várias medições ( $\mathrm{E}$. JUNIOR; GOMES; SILVA, 2010).

Para viabilização do estudo foram realizadas várias medições para coleta dos dados e posterior análise através do software gratuito NetStumbler. Também foi utilizado um GPS de modelo GARMIN ETREX LEGEND, para a localização geográfica dos pontos medidos no estacionamento, além de um laptop modelo TOSHIBA Satelitte Pro A200, com uma antena interna com ganho de $4.8 \mathrm{dBi}$.

Após a instalação do software NetStumbler no notebook e a conexão do mesmo com o GPS, foi realizada a captação dos dados, os quais foram posteriormente tratados e mapeados com base na planta baixa do local, como mostra a Figura 4 .

As etapas desse levantamento foram:

A. Demarcação dos pontos de medição;

B. Análise dos pontos com o NetStumbler,

C. Tratamento no Excel dos dados coletados;

D. Plotagem da planta baixa com demarcação dos pontos;

E. Apresentação dos dados no software Surfer. 


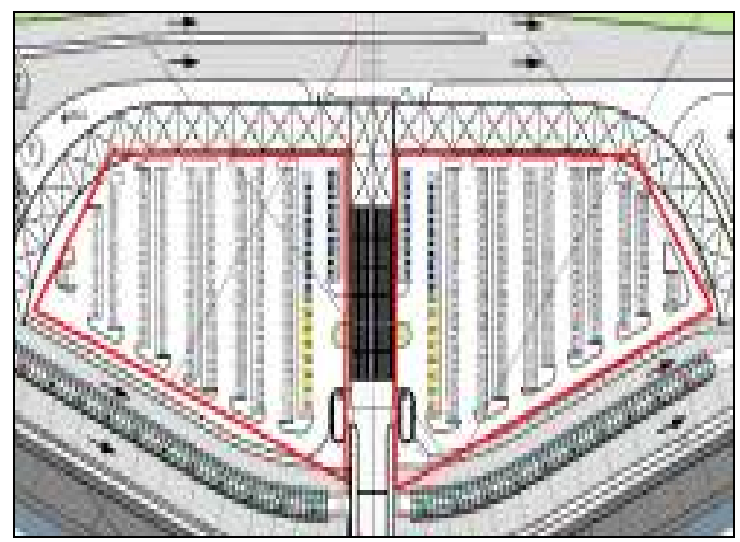

FIGURA 4. Planta baixa do estacionamento com a área da coleta dos dados demarcada em vermelho.

FONTE: INFRAERO, 2011.

As próximas seções descrevem cada uma dessas etapas.

\section{A. DemarcaÇÃo dos PONTOS}

Para realizar a coleta dos pontos na área de medição foi adotado um critério de leitura para cada vaga do estacionamento, com a distância entre um ponto e outro de 2,5 metros, cobrindo assim todas as 395 vagas do estacionamento. Foram coletados ao todo 395 pontos. Após a demarcação, os pontos foram validados para que suas posições correspondessem exatamente ao mundo real. A Figura 5 mostra como foi feita a coleta de dados.



FIGURA 5. Imagem demonstrando o momento da coleta de dados em uma das vagas do estacionamento.

\section{B. AnÁlise dos pontos com NetStumbler}

Após a seleção, demarcação e medição dos pontos foi iniciada a análise de cada ponto individualmente utilizando o software NetStumbler instalado no notebook.

Inicialmente foi realizada uma medição no lado esquerdo do estacionamento (entrada de veículos) para a verificação da qualidade do sinal da antena painel direcionada para aquele local.

Após o início das medições, o software NetStumbler localizou o Ponto de Acesso que se encontra no raio de alcance da antena do notebook, coletando dados como potência do sinal, ruído e relação sinal/ruído através de um gráfico exibido durante a coleta dos dados, conforme mostra a Figura 6. Estes dados foram exportados em formato txt (texto) e, posteriormente, convertidos para o formato .x/s (Microsoft Excel) onde foram tratados.

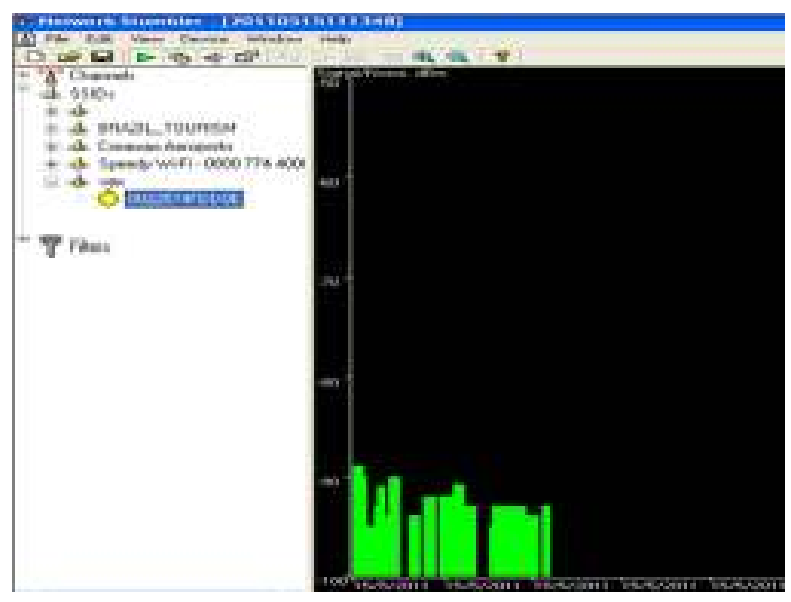

FIGURA 6. Gráfico gerado pelo NetStumbler durante a coleta dos dados.

A análise foi realizada no ambiente, com o notebook no estado estacionário em cada vaga do estacionamento. O SSID localizado pelo NetStumbler para as antenas painéis direcionadas para o estacionamento foi o Vex.

O SSID é um conjunto único de caracteres que identifica uma rede sem fio, ou seja, é basicamente o 
"nome" dado à rede. Para este SSID foram identificados dois endereços MAC: 002369B3D201 e 0022574FDD0E. O MAC 002369B3D201 corresponde ao lado esquerdo do estacionamento (entrada de veículos) e o MAC 0022574FDD0E ao lado direito do mesmo (saída de veículos).

\section{C. tratamento no Excel dos dados COLETADOS}

Durante a visualização dos dados, isolou-se a coluna que informa os valores referentes ao sinal (SIG) e também as colunas que informam as coordenadas geográficas de cada ponto (Longitude e Latitude). A Figura 7 demonstra como foi feito o tratamento dos dados.

\begin{tabular}{|c|c|c|c|c|c|c|}
\hline  & A & B & C & 0 & $\mathrm{E}$ & $F$ \\
\hline 1 & \multicolumn{2}{|c|}{ "SCreator: Networlf } & \multicolumn{2}{|l|}{ Stumbler Version 0.4.0 } & & \\
\hline 2 & \multicolumn{2}{|c|}{ ISFormat: wi-scen } & thestensions & & & \\
\hline 3 & \multicolumn{2}{|c|}{ \# SDateGMT: 2011-0 } & -15 & & & \\
\hline \multicolumn{7}{|l|}{4} \\
\hline$s$ & {$[$ SSID $]$} & Tyos & (BSSO) & [SNA Sig Noise] & Finame $]$ & Flap: \\
\hline 6 & $(v e x)$ & GSS & [00:22:57.4t:dd:06 \} & [95849] & $\approx(1)$ & 421 \\
\hline 7 & (vex) & GSS & [00:22:57:4f:dd:0e \} & [95849] & $\approx()$ & 421 \\
\hline 8 & $\langle$ vex $\rangle$ & BSS & [00:22:57:4f:dd:0s] & [95849] & $\approx(1)$ & 421 \\
\hline 9 & (vex) & BSS & [00:22:57:4f:dd:0s] & [95849] & $\approx(1)$ & 421 \\
\hline 10 & $\langle$ vex $)$ & BSS & [00:22:57:4f:dd:0s] & [95849] & $\approx(1)$ & 421 \\
\hline 11 & $\langle$ vex $\rangle$ & BSS & [00:22:57.4t:dd:0e \} & {$[75649]$} & $\approx()$ & 421 \\
\hline 12 & (vex) & BSS & [00:22:57.4f:dd:0s] & [75649] & $*()$ & 421 \\
\hline 13 & (vex) & BSS & [00:22:57:4t:dd:0s] & {$[75649]$} & $\approx()$ & 421 \\
\hline 14 & $\langle$ vex $)$ & BSS & [00:22:57:4f:dd:0s] & {$[75649]$} & $\approx(1)$ & 421 \\
\hline 15 & $($ vex $)$ & BSS & [00:22:57.4t:dd:0s] & [75649] & $\approx(1)$ & 421 \\
\hline 16 & $($ vex $)$ & BSS & [00:22:57:4f:dd:0s] & {$[75649]$} & $\approx(1)$ & 421 \\
\hline 17 & (vex) & BSS & [00:22:57:4t:dd:0s] & [75649] & $\approx(1)$ & 421 \\
\hline 18 & (vex) & GSS & [00:22:57:4t:dd:0s] & {$[75649]$} & $\approx(1)$ & 421 \\
\hline
\end{tabular}

FIGURA 7. Tratamento dos dados coletados no MSExcel

Posteriormente, foi feita a conversão de $\mathrm{dBm}$ (decibel miliwatt - medida de potência de um equipamento de transmissão) para miliWatt. Em seguida, calculou-se a média e converteu-se novamente de miliwatt para $\mathrm{dBm}$.

Após os dados medidos terem sido tratados, foi criada uma nova planilha com os valores referentes à coordenada $X$ do ponto coletado (Latitude), os valores referentes à coordenada $Y$ (Longitude) e os valores da média da potência de sinal em $\mathrm{dBm}$.

\section{Plotagem da planta baixa com DEMARCAÇÃO DOS PONTOS}

Após a formatação final dos dados no Excel, foi realizada a digitalização dessas informações através do software AutoCad.

Os dados coletados foram inseridos no Autocad e georreferenciados sobre o mapa base da área (planta baixa do estacionamento). Após plotado o desenho no AutoCAD, o documento digitalizado foi salvo no formato .dxf e guardado para posterior cruzamento com os dados a serem gerados pelo software Surfer.

\section{E. Apresentação dos dados no software Surfer}

Depois dos dados terem sido tratados e devidamente mapeados na planta baixa do estacionamento, foi utilizado o software Surfer versão 8.0 para criar o zoneamento da intensidade do sinal no estacionamento analisado, criando o gráfico da propagação do sinal e detectando os pontos onde o sinal é deficiente ou mesmo até ausente, podendo, assim, analisar os pontos fortes e fracos da intensidade do sinal no local estudado.

O cruzamento dos dados no Surfer foi realizado através da planta baixa, previamente digitalizada no AutoCAD, no qual pode ser adicionada à plotagem através do menu "Map/Base Map".

A planilha do Excel que contêm as informações das coordenadas topográficas de cada ponto coletado, bem como as médias de intensidade de sinal captadas através do NetStumbler foram inseridas no Surfer através do menu "Grid/Data".

Em seguida, o método para interpolação dos pontos foi escolhido, acessando o menu "Gridding Method" através da opção "Triangulation with Linear Interpolation". Após este procedimento, um arquivo .grd (grid) foi exportado pelo Surfer e, posteriormente, importado pelo mesmo programa através do menu

e-xacta, Belo Horizonte, v. 4, n.1, p. 103-109. (2011) Editora UniBH Disponível em: www.unibh.br/revistas/exacta/ 
"Open Grid". Em seguida, o gráfico foi finalmente plotado como exposto na Figura 8.



FIGURA 8. Mapa de zoneamento do sinal Wi-Fi correspondente ao MAC 002369B3D201.

\section{Resultados E discussão}

Após a análise dos pontos, o tratamento dos dados e a criação do zoneamento de intensidade do sinal Wi$F i$, pode-se observar na Figura 9 o maior nível de potência recebido, representado em vermelho e o menor nível de potência recebido, representado em azul.



FIGURA 9. Planta baixa com os mapas de zoneamento de intensidade do sinal indicando a localização das antenas painéis na torre frontal (ícone).
Observando a escala de intensidade do sinal presente na Figura 9, pode-se observar que a região central do estacionamento foi o local onde se obteve os melhores resultados para uma melhor conexão de dados. Esse fato está intimamente relacionado ao tipo de antena utilizada (antena painel) na geração do hotspot, já que é necessário um sinal de pelo menos $92 \mathrm{dBm}$ para manter uma conexão na velocidade mínima (1 megabit) e pelo menos $-72 \mathrm{dBm}$ para manter uma conexão a 54 megabits.

As antenas painéis, instaladas pela Infraero, foram utilizadas de forma proposital na infra-estrutura do estacionamento afim de não permitir a evasão do hotspot para as áreas de embarque e da torre de comando do aeroporto, evitando assim possíveis interferências nas áreas em questão.

\section{CoNCLUSÃo}

Foi apresentado neste artigo uma técnica para análise de sinais Wi-Fi oriundas de equipamentos de redes wireless em ambiente outdoor, cujo objetivo era estudar a metodologia Site Survey através de um estudo de caso no estacionamento do Aeroporto Internacional Tancredo Neves e apresentar, através de gráficos e informações coletadas na área, os resultados e discussões sobre este trabalho.

Através deste estudo, conclui-se que o Site Survey é uma técnica que pode ser utilizada para a análise da propagação das ondas de rádio em ambientes outdoor, sendo uma ferramenta que disponibiliza as informações necessárias a avaliação de uma rede wireless.

FARIAS, P. C. - Curso Essencial em Redes. Ed. Digerati Books, 2007 - 160p. 
GOOGLEMAPS - Google do Brasil - 2011. Disponível em: http://maps.google.com.br/Aeroporto Internacional Tancredo Neves. Acesso em: 1 abr. 2011.

INFRAERO - Empresa Brasileira de Infraestrutura Aeroportuária - Apresenta textos e mapas sobre os aeroportos brasileiros. Disponível em: http://www.infraero.gov.br - Acesso em: 1 maio 2011.

JARDIM, F. M. - Treinamento Avançado em Redes Wireless. Ed. Digerati Books, 2007 - 128p.

JUNIOR, E.S.C.; GOMES, P.V.P; SILVA, F.C.M.; Análise da propagação de sinais de rádio para implantação de uma WLAN. Disponível em: http://www3.iesam-pa.edu.br/ojs/index.php/

sistemas/article/view/490/392. Acesso em: 12 abr. 2011.

LINKTECK - LinkTeck Wireless Distribuidora do Brasil - Disponível em: http://www.linkteck.com.br/lojanova/produtos /detalhe.php?codigo_produto=1206. Acesso em: 10 maio 2011.

PINHEIRO, J. M.- A Trilogia Wireless -Malima Consultoria, 2003 - Disponível em: www.malima.com.br/article_read.asp?id=22 Acesso: em 15 fev. 2011.

VAUGHAN-NICHOLS, S. J. "Are Site Surveys Unnecessary?", 2003 - Disponível em: http://www.wifiplanet.com/tutorials/article.php /2243911> - Acesso em: 5 mar. 2011.

WORDPRESS, wordpress.com - Aeroporto de Confins - Site de informações sobre o aeroporto de Confins. Disponível em: http://aeroportodeconfins.wordpress.com/ Acesso em: 1 maio 2011. 